THE ASTROPHYSICAL JOURNAL, 2009, IN PRESS

Preprint typeset using LATEX style emulateapj v. 03/07/07

\title{
EMPIRICAL CONSTRAINTS ON PROTON AND ELECTRON HEATING IN THE FAST SOLAR WIND
}

\author{
Steven R. Cranmer ${ }^{1}$, William H. Matthaeus ${ }^{2}$, Benjamin A. Breech ${ }^{3}$, And Justin C. Kasper ${ }^{1}$ \\ Draft version October 14, 2018
}

\begin{abstract}
We analyze measured proton and electron temperatures in the high-speed solar wind in order to calculate the separate rates of heat deposition for protons and electrons. When comparing with other regions of the heliosphere, the fast solar wind has the lowest density and the least frequent Coulomb collisions. This makes the fast wind an optimal testing ground for studies of collisionless kinetic processes associated with the dissipation of plasma turbulence. Data from the Helios and Ulysses plasma instruments were collected to determine mean radial trends in the temperatures and the electron heat conduction flux between 0.29 and 5.4 AU. The derived heating rates apply specifically for these mean plasma properties and not for the full range of measured values around the mean. We found that the protons receive about $60 \%$ of the total plasma heating in the inner heliosphere, and that this fraction increases to approximately $80 \%$ by the orbit of Jupiter. A major factor affecting the uncertainty in this fraction is the uncertainty in the measured radial gradient of the electron heat conduction flux. The empirically derived partitioning of heat between protons and electrons is in rough agreement with theoretical predictions from a model of linear Vlasov wave damping. For a modeled power spectrum consisting only of Alfvénic fluctuations, the best agreement was found for a distribution of wavenumber vectors that evolves toward isotropy as distance increases.
\end{abstract}

Subject headings: hydrodynamics - MHD — plasmas — solar wind — turbulence — waves

\section{INTRODUCTION}

The supersonic solar wind is accelerated away from the Sun by some combination of physical processes including gradients in gas pressure (from the hot, $10^{6} \mathrm{~K}$ corona) and wave pressure, as well as possible collisionless wave-particle interactions. There is gradual heat input into the solar wind plasma that begins in the corona and extends far out into interplanetary space (see reviews by Parker 1963; Leer et al. 1982; Tu \& Marsch 1995; Goldstein et al. 1995; Marsch 1999; Hollweg \& Isenberg 2002; Cranmer 2002; Matthaeus et al. 2003). One likely source of this extended heating is the dissipation of magnetohydrodynamic (MHD) turbulence. In order to better understand the combined problem of coronal heating, solar wind acceleration, and the large-scale evolution of the turbulent heliospheric plasma, we need to know how energy is transferred between the MHD fluctuations and the particles.

In the mainly collisionless solar wind, the various particle species (i.e., protons, electrons, and heavy ions) are not in thermal equilibrium with one another. The particles exhibit a range of different outflow speeds, temperatures, and velocity distribution anisotropies, and these differences are most pronounced in low-density regions with the least frequent Coulomb collisions (e.g., Neugebauer 1982; Kohl et al. 1997, 1998, 2006; Kasper et al. 2008). These differences can be used to probe the kinetic physical processes that are responsible for depositing energy into the plasma.

There have been a number of studies where the measured plasma properties in the solar wind were used to derive the corresponding rates of energy input from processes such as MHD turbulence (e.g., Coleman 1968; Tu 1988; Freeman 1988; Verma et al. 1995; Matthaeus et al. 1999b; Smith et

\footnotetext{
${ }^{1}$ Harvard-Smithsonian Center for Astrophysics, 60 Garden Street, Cambridge, MA 02138

2 Department of Physics and Astronomy, Bartol Research Institute, University of Delaware, Newark, DE 19716

${ }^{3}$ NASA Goddard Space Flight Center, 8800 Greenbelt Road, Greenbelt, MD 20771
}

al. 2001; Vasquez et al. 2007; MacBride et al. 2008; Marino et al. 2008; Breech et al. 2008). Much of this work, however, dealt only with the energy budget of the protons and not with any other ions or the electrons. Although the solar wind's mass density and momentum flux are dominated by the protons, the electrons carry approximately half of the thermal energy of the plasma and should not be neglected in a complete treatment. There have been other investigations into the electron energy balance in the solar wind (e.g., Scudder \& Olbert 1979; Phillips \& Gosling 1990; Pilipp et al. 1990). There also have been studies of turbulent dissipation that compare a total heating rate to the presumed proton contribution-and thus estimate the electron heating rate as the remainder (Leamon et al. 1999; Stawarz et al. 2009). However, there has been surprisingly little combined analysis of the proton and electron heating rates that treat the two plasma components on equal footing.

In this paper we compute new estimates of proton and electron heating rates in the fast solar wind. These rates are derived from plasma properties measured by the Helios and Ulysses spacecraft. In $\S 2$ we describe these measurements in detail, and in $\S 3$ we show how the heating rates can be computed from the separate equations of energy conservation for protons and electrons. We find, not surprisingly, that an accurate determination of the electron heating rate depends crucially on the measured electron heat conduction flux. In $\S 4$ we compare the empirically derived heating rates with heating rates obtained from linear Vlasov theory, in which several simple assumptions about the symmetry of an (Alfvénic) fluctuation spectrum were made. Finally, $\S 5$ contains a summary of the major results of this paper and a discussion of the implications these results may have on our wider understanding of heliospheric plasma physics.

This work is being presented in tandem with an independent investigation by Breech et al. (2009). In both papers, we studied the same problem of proton-electron heat partitioning in the fast wind, but it has been approached from different and complementary vantage points. This paper attempts 
to "invert" the in situ measurements in order to determine the partition fraction between proton and electron heating. On the other hand, Breech et al. (2009) showed how a sophisticated model of MHD turbulent heating - with an assumed value for the partition fraction - can also be consistent with the empirical data. The results of these two studies are consistent with one another.

\section{IN SITU PARTICLE DATA}

We examine proton and electron plasma properties for the high-speed solar wind between 0.29 and 5.4 AU. Measurements made at larger distances (e.g., from the Voyager probes) are excluded for two reasons: (1) these data have been taken mainly in the ecliptic plane, and thus are dominated by slowspeed wind, and (2) the internal energy budget of particle velocity distributions at distances $r \gtrsim 10$ AU is increasingly affected by pickup ions, which are ignored here. We focus on fast wind streams because these appear to be the sites of the most ambient and "quiescent" solar wind plasma, and because their low densities highlight them as the least complicated by inter-species collisional coupling.

Figure 1 displays the data we use for the proton temperature $T_{p}$, electron temperature $T_{e}$, and electron parallel heat conduction flux $q_{\|, e}$. Operationally, the high-speed wind was defined as being all streams faster than $600 \mathrm{~km} \mathrm{~s}^{-1}$. This selection criterion was used for both the Helios and Ulysses data sets. A speed of $600 \mathrm{~km} \mathrm{~s}^{-1}$ is slightly more restrictive than the more standard value of $500 \mathrm{~km} \mathrm{~s}^{-1}$ that has been applied by others to define the fast wind (e.g., Dasso et al. 2005; MacBride et al. 2008). Taking a narrower range of speeds results in less contamination from plasma parcels with qualitatively different properties.

All measurements for heliocentric distances $r$ less than 1 AU came from published data from the plasma instruments on the two Helios spacecraft (Schwenn \& Marsch 1990). Proton temperatures for the fast wind were obtained from Marsch et al. (1982) and electron plasma properties $\left(T_{e}\right.$ and $\left.q_{\|, e}\right)$ were taken from Pilipp et al. (1990). The tabulated data points, which spanned the near-solar-minimum years 19741976 , corresponded to representative intervals that were selected for broad coverage in distance and wind speed. The actual velocity distributions for both protons and electrons were non-Maxwellian, and the temperatures used here are mean isotropic (drifting Maxwellian) equivalents, i.e., $T \equiv$ $\left(T_{\|}+2 T_{\perp}\right) / 3$.

Data for $r>1$ AU came from the "Solar Wind Observations Over the Poles of the Sun" (SWOOPS) instrument on Ulysses (Bame et al. 1992; Goldstein et al. 1996). The actual temperature measurements were extracted from the European Space Agency's online archive. ${ }^{4}$ We collected all SWOOPS data for the decade of time starting at the beginning of the Ulysses mission (1990 November) and extending to 2000 December. This fully encompassed the solar minimum in 1996-1997, during which there were found to be large swaths of the heliospheric volume containing quiescent fast solar wind. The highest time resolution data available for each interval was used to better pinpoint regions of high-speed wind (using the proton outflow speed $u_{p}$ ). For the proton temperature data, this corresponded to a 4 or 8 minute cadence, depending on the mode of operation. For the electron temperatures, the highest cadence tended to vary between 7 and 35 minutes.

4 The ESA Ulysses Data System (UDS) site for SWOOPS data is: http://helio.esa.int/ulysses/archive/swoops.html
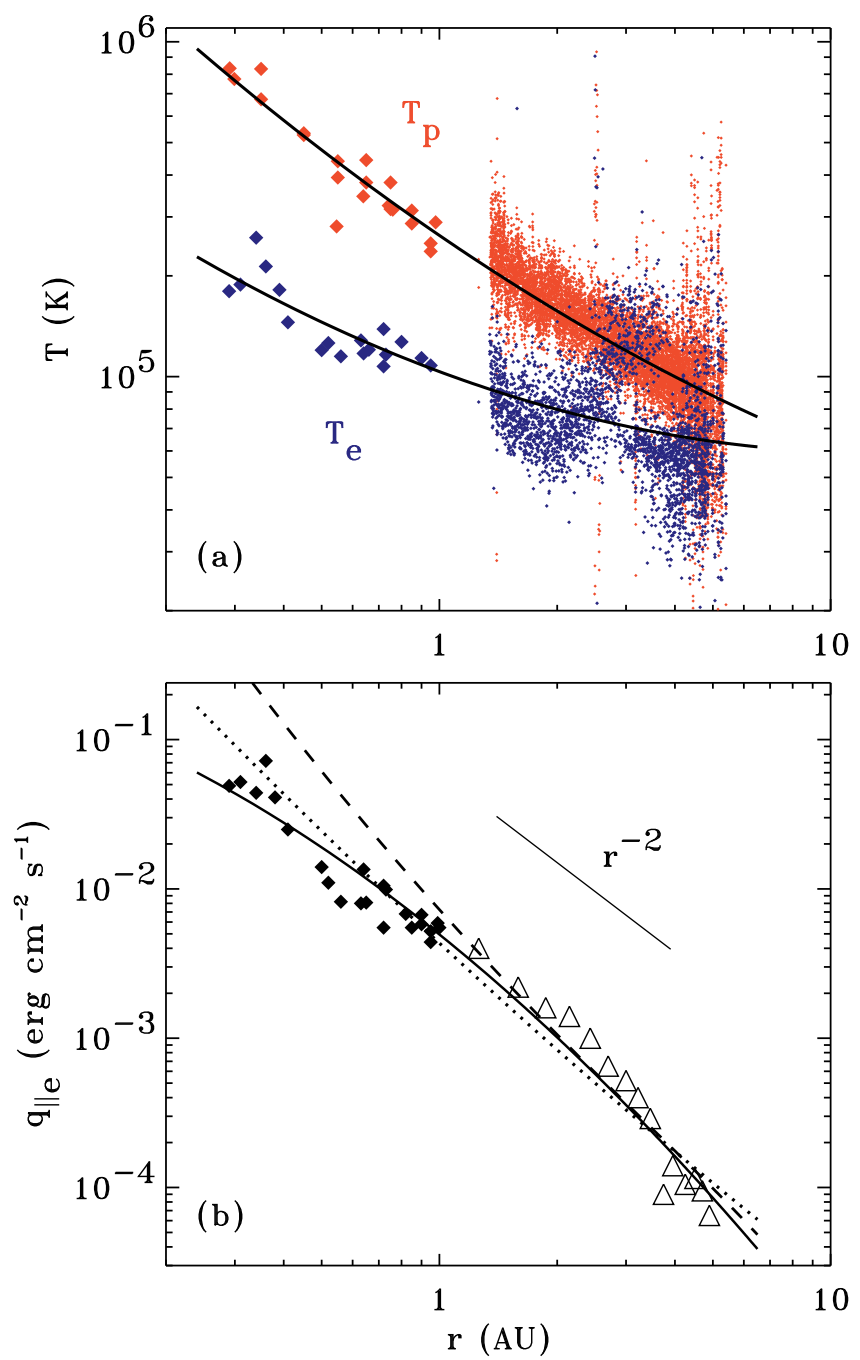

FIG. 1.- In situ measurements for the fast solar wind: (a) plasma temperatures from Helios (filled diamonds) and Ulysses (small points), with protons in red and electrons in blue. (b) electron heat conduction flux from Helios (filled diamonds) and Ulysses (triangles). Also shown are least-squares fits (solid lines), the classical Spitzer-Härm heat flux (dashed line), and the Hollweg $(1974,1976)$ collisionless heat flux with $\alpha_{e}=1.05$ (dotted line).

The Ulysses electron heat conduction data shown in Figure $1 b$ were taken from Figure 7 of Scime et al. (1994). These measurements were taken in the ecliptic plane in 1990-1992 during the initial Ulysses cruise phase from 1 to $5 \mathrm{AU}$. These data were not provided in a format where the high-speed wind selection criterion $\left(u_{p} \geq 600 \mathrm{~km} \mathrm{~s}^{-1}\right)$ could be applied. Thus, the points in Figure $1 b$ at $r>1$ AU apply to all wind speed intervals. However, Scime et al. (1999) found that there was no significant variation in $q_{\|, e}$ as Ulysses passed between fast and slow wind streams during its first high-latitude scan in 1994-1995 (see also Salem et al. 2003).

There is a drastic difference in the number of data points shown in Figure $1 a$ between the Helios and Ulysses measurements. For Helios, the high-speed velocity criterion resulted in only 21 data points for $T_{p}$ and 18 data points for $T_{e}$. For Ulysses, however, there were $211593 T_{p}$ data points and $61865 T_{e}$ data points for the fast wind. This is actually only a subset of the total amount of data present in the archive, since we used only proton data validated by a quality control flag that indicated nothing detectably wrong with the measurement. In order to keep Figure 1 from being unreason- 
ably large in size, we plotted every 20th individual SWOOPS temperature measurement. For the collection of Ulysses data (chosen by the criterion of $u_{p} \geq 600 \mathrm{~km} \mathrm{~s}^{-1}$ ), the mean wind speed was found to be $744 \mathrm{~km} \mathrm{~s}^{-1}$, with a standard deviation about this mean of $47 \mathrm{~km} \mathrm{~s}^{-1}$. If the data selection criterion is reduced to $u_{p} \geq 500 \mathrm{~km} \mathrm{~s}^{-1}$, the mean speed and standard deviation change to $705 \mathrm{~km} \mathrm{~s}^{-1}$ and $92 \mathrm{~km} \mathrm{~s}^{-1}$, respectively.

There are a number of potential difficulties and systematic uncertainties in measuring $T_{p}$ with the Ulysses plasma instrument. Some of these issues are more problematic for the slow wind, since these regions tend to have lower values of $T_{p}$ and thus narrower distribution functions in velocity space. These measurements may suffer from discretization effects caused by the finite number of energy and angle channels of the SWOOPS instrument. However, like many solar wind instruments, SWOOPS has a logarithmic distribution in the spacing of its energy channels. This could result in an additional source of error for the fast wind, since the velocity distributions are "centered" at higher speeds where the relative energy resolution is coarser and the protons are thus spread across fewer energy windows.

In order to convey a sense of the measurement uncertainties, the SWOOPS data archive reports two independent determinations of the proton temperature that are claimed to usually bracket the true proton temperature from below $\left(T_{\text {small }}\right)$ and from above $\left(T_{\text {large }}\right)$ :

1. The lower limit value $T_{\text {small }}$ is essentially just the radial component of the proton temperature, with the high-energy "beam" component cut off. Goldstein et al. (1996) concluded that the proton velocity distribution is roughly isotropic-over the Ulysses distancesbecause there was never a substantial dependence of the radial temperature on the local magnetic field direction (see also Vasquez et al. 2007). Thus, the closer the distribution tends to being isotropic in velocity space, the better an assumption $T_{\text {small }}$ will be.

2. The upper limit value $T_{\text {large }}$ was obtained by integrating the measured proton velocity distribution over all of the energy channels and angle bins that were statistically above a determined noise level. For the lowest temperatures, this process results in an overestimate of $T_{p}$ because the angular responses of the SWOOPS instrument channels may be broader than the actual velocity distribution function.

In Figure $1 a$, we adopt the geometric mean of these two bounding values to obtain an intermediate estimate for $T_{p}=$ $\left(T_{\text {small }} T_{\text {large }}\right)^{1 / 2}$. It should be noted that there seems to be a solar cycle trend in the ratio $T_{\text {large }} / T_{\text {small }}$. At solar maximum (i.e., around 1990 and 2000), this ratio tends to range between 2 and 3. At solar minimum (i.e., 1996-1997), the ratio tends to range between only 1.2 and 1.5 . Thus, the more we depend on the solar minimum SWOOPS data (see below), the less we need to be concerned about the differences between $T_{\text {large }}$ and $T_{\text {small }}$ (see also Marino et al. 2008).

For the Ulysses electron data, we used the total temperatures formed by a weighted sum of the narrow thermal core and the broader "halo" and "strahl" components of the velocity distributions. The total electron temperature is the most consistent quantity to use with the internal energy moment equations described in $\S 3.1$, since these equations were derived by integrating over all velocity space. Figure $1 a$ shows a noticeable bifurcation of $T_{e}$ into hot and cold branches at distances around $r \approx 3-4$ AU. This appears to be a solar cycle effect, since the lower values are more dominant during 1996 (solar minimum) and the higher values are more favored during the two solar maximum periods (1991 and 2000) and, to a lesser extent, the post-maximum phase (1994). The higher $T_{e}$ data points at 3-4 AU also exhibited slightly higher electron densities $n_{e}$ than the cooler data points. Thus, the lower values seem more appropriate to be applied to studies of the ambient fast solar wind associated with polar coronal holes at solar minimum. The least squares fit curve shown in Figure $1 a$ for $T_{e}$ serendipitously favors these lower values.

Figure 1 also shows analytic fits for the measured quantities between 0.29 and 5.4 AU. These fits are given by

$$
\begin{aligned}
\ln \left(\frac{T_{p}}{10^{5} \mathrm{~K}}\right) & =0.9711-0.7988 x+0.07062 x^{2} \\
\ln \left(\frac{T_{e}}{10^{5} \mathrm{~K}}\right) & =0.03460-0.4333 x+0.08383 x^{2} \\
\ln \left(\frac{q_{\|, e}}{q_{0}}\right) & =-0.7032-2.115 x-0.2545 x^{2}
\end{aligned}
$$

where $x \equiv \ln (r /[1 \mathrm{AU}])$ and $q_{0}=0.01 \mathrm{erg} \mathrm{cm}^{-2} \mathrm{~s}^{-1}$. To avoid the larger number of Ulysses data points overwhelming the Helios data points in the least-squares fitting process, the data sets from the two spacecraft were weighted equally. These fits should not be extended very far beyond the range of heliocentric distances $(0.29<r<5.4 \mathrm{AU})$ for which they were derived.

The new fits given above can be analyzed in terms of a commonly assumed power-law dependence of temperature as a function of radius; i.e., $T \propto r^{-\delta}$. The local value of the exponent $\delta$ can be computed at any distance as the logarithmic derivative $-\partial \ln T / \partial \ln r$. Using equations (1) and (2), the resulting proton and electron temperature exponents $\delta_{p}$ and $\delta_{e}$ both decrease monotonically with increasing distance. For protons, $\delta_{p}$ is approximately 0.98 at $0.3 \mathrm{AU}$, it decreases to 0.80 at $1 \mathrm{AU}$, and then to 0.56 at $5 \mathrm{AU}$. This range of values agrees with other exponents reported over these distances that range between about 0.75 and 1 (e.g., Eyni \& Steinitz 1981; Lopez \& Freeman 1986; Totten et al. 1995; Ebert et al. 2009). For electrons, $\delta_{e}$ is approximately 0.65 at $0.3 \mathrm{AU}$, it decreases to 0.43 at $1 \mathrm{AU}$, and to 0.15 at $5 \mathrm{AU}$. These are also in agreement with earlier measurements of order 0.2-0.6 (see Sittler \& Scudder 1980; Pilipp et al. 1990; Phillips et al. 1995; Issautier et al. 1998). Some of these studies did not include any explicit criteria for selecting either fast or slow solar wind streams, but it is interesting that the same rough range of exponents is found. It is possible to use these exponents to derive an effective polytropic index for the protons and electrons-i.e., $\gamma=1+(\delta / 2)$ (Totten et al. 1995)-but the analysis in $\S 3$ attempts to do a more thorough study of the internal energy balance of the plasma.

Figure $1 b$ also shows how the measured electron heat conduction flux compares to both the classical collisional heat flux (Spitzer \& Härm 1953) and to Hollweg's (1974, 1976) collisionless "free streaming" approximation. The former is given by

$$
q_{\|, e}=-\kappa_{e} \frac{\partial T_{e}}{\partial r}
$$

where the electron conductivity is

$$
\kappa_{e}=\left(1.84 \times 10^{-5} \mathrm{erg} \mathrm{cm}^{-1} \mathrm{~s}^{-1} \mathrm{~K}^{-7 / 2}\right) \frac{T_{e}^{5 / 2}}{\ln \Lambda_{e e}} .
$$


Also, the electron Coulomb logarithm is approximated by

$$
\ln \Lambda_{e e}=23.2+\frac{3}{2} \ln \left(\frac{T_{e}}{10^{6} \mathrm{~K}}\right)-\frac{1}{2} \ln \left(\frac{n_{e}}{10^{6} \mathrm{~cm}^{-3}}\right)
$$

where $n_{e}$ is the electron number density (see also Cranmer et al. 2007). The Spitzer-Härm values are reasonably close to the measured data above $r \approx 1 \mathrm{AU}$, but they exceed the measurements at smaller distances. The Hollweg (1974, 1976) heat flux is given by

$$
q_{\|, e} \approx \frac{3}{2} \alpha_{e} n_{e} u k_{\mathrm{B}} T_{e},
$$

where $k_{\mathrm{B}}$ is Boltzmann's constant. The dimensionless orderunity constant $\alpha_{e}$ is only known approximately, and it is expected to depend on the microscopic shape of the electron velocity distribution (Hollweg 1974, 1976). Using the above expression, though, we solved for $\alpha_{e}$ using each of the data points in Figure $1 b$. For simplicity, we used the above fit for $T_{e}(r)$, we also assumed that $u=700 \mathrm{~km} \mathrm{~s}^{-1}$, and we used the empirical analytic model for $n_{e}(r)$ that is described below. The mean value of $\alpha_{e}$ for all 38 points was 1.05 , with a standard deviation about the mean of 0.44 and no clear radial trend. (The full set of values ranged between 0.47 and 2.08.) For comparison, Figure $1 b$ shows a corresponding curve for the collisionless heat conduction flux when $\alpha_{e}=1.05$.

\section{EMPIRICAL HEATING RATES}

\subsection{Internal Energy Conservation Equations}

Our goal is to use measurements to quantify the rates of heating and cooling associated with as many physical processes as possible, and then to solve for the net volumetric rates of heat input that presumably can be attributed to MHD turbulence. The primary mechanisms that are taken into account include adiabatic energy conservation, electron heat conduction, and Coulomb collisions. We also retain the assumption that both the proton and electron velocity distributions are isotropic Maxwell-Boltzmann distributions with a shared bulk velocity $\left(u_{p}=u_{e}\right)$. Direct collisions between the proton and electron populations are included for completeness, but they are expected to be extremely weak in the heliosphere. The models computed using classical collision rates (e.g., Spitzer \& Härm 1953) are virtually identical to those without any collisions whatsoever. However, we also study the ramifications of more rapid collisions by scaling up the classical rates by a range of constant factors.

It is important to discuss some of the physical processes that are neglected in the models presented below. For example, we do not explicitly consider how departures from temperature isotropy (i.e., $T_{\|} \neq T_{\perp}$ ) affect the energy balance. Observationally, these departures are not significantly large over most of the distances considered here (see Marsch et al. 1982; Pilipp et al. 1990; Matteini et al. 2007). At 1 AU, Vasquez et al. (2007) showed that taking account of the measured range of anisotropies would result in, at most, about a $10 \%$ change in the adiabatic terms of the energy conservation equations. Still, it has been shown that for some regions in the solar wind, the velocity distributions are kept from deforming too far away from isotropy by plasma instabilities (e.g., Kasper et al. 2002; Hellinger et al. 2006). We defer any consideration of the energy transfer between instability-generated waves and the particles to future work.

Another process that is neglected here is proton heat conduction. The classical proton heat conduction coefficient is about 25 times smaller than that for electrons (Braginskii 1965; Sandbæk \& Leer 1995). Also, some models based on higher-order moment closures of the Boltzmann equation have found even lower values than the classical approach would suggest (e.g., Olsen \& Leer 1996). The proton heat conduction flux was measured by Helios between 0.3 and 1 AU (Marsch et al. 1982). To confirm the expectation that the proton heat flux can be neglected, we analyzed these measurements in terms of the collisionless free-streaming approximation discussed above. In other words, for each measurement we computed an effective coefficient $\alpha_{p}$ analogous to the electron coefficient $\alpha_{e}$ in equation (7). Using the data tabulated by Marsch et al. (1982), we obtained a mean value of $\alpha_{p}=0.045$, which is a factor of $\sim 23$ lower than the mean electron coefficient $\alpha_{e}=1.05$. Test runs of the energy conservation models that included this level of proton heat conduction resulted in heating rates that were within $5 \%$ of the ones computed without this effect.

We are now in a position to describe the conservation laws for proton and electron internal energy upon which the present results will be based. Assuming a time-steady solar wind that has reached a constant asymptotic terminal speed $u$ (see, e.g., Arya \& Freeman 1991), these equations are

$$
\begin{gathered}
\frac{3}{2} n_{p} u k_{\mathrm{B}} \frac{\partial T_{p}}{\partial r}-u k_{\mathrm{B}} T_{p} \frac{\partial n_{p}}{\partial r}=Q_{p}+\frac{3}{2} n_{p} k_{\mathrm{B}} \nu_{p e}\left(T_{e}-T_{p}\right) \\
\frac{3}{2} n_{e} u k_{\mathrm{B}} \frac{\partial T_{e}}{\partial r}-u k_{\mathrm{B}} T_{e} \frac{\partial n_{e}}{\partial r}=Q_{e}+\frac{3}{2} n_{e} k_{\mathrm{B}} \nu_{e p}\left(T_{p}-T_{e}\right) \\
-\frac{1}{r^{2}} \frac{\partial}{\partial r}\left(q_{\|, e} r^{2} \cos ^{2} \Phi\right)
\end{gathered}
$$

where the heat input rates are $Q_{p}$ and $Q_{e}$, the Parker spiral angle is $\Phi$, and the rates of proton-electron Coulomb collisions for the two equations are $\nu_{p e}$ and $\nu_{e p}$ (see Barakat \& Schunk 1982; Isenberg 1984; Cranmer et al. 1999). As mentioned above, we assumed that the outflow speed $u$ is constant and identical for the protons and electrons. We also assumed that the electron densities $n_{e}$ and $n_{p}$ vary with distance as $r^{-2}$, and that $n_{p}$ is normalized to a value of $2.5 \mathrm{~cm}^{-3}$ at $1 \mathrm{AU}$ (e.g., Goldstein et al. 1996). We utilized a 5\% helium abundance by number in order to compute $n_{e}=1.1 n_{p}$. Also, the winding angle of the spiral interplanetary magnetic field is given in its standard form as

$$
\tan \Phi=\Omega r \sin \theta / u
$$

where we used a rotation frequency $\Omega=2.7 \times 10^{-6} \mathrm{rad} \mathrm{s}^{-1}$. In most of the models shown below, we set the colatitude $\theta=15^{\circ}$ to model the high-latitude Ulysses measurements. ${ }^{5}$

The Coulomb collision frequencies are balanced such that $n_{e} \nu_{e p} \approx n_{p} \nu_{p e}$. For two isotropic Maxwellian distributions interacting with one another,

$$
n_{p} \nu_{p e}=\frac{32}{3} \pi^{1 / 2} \ln \Lambda \frac{e^{4} n_{e} n_{p}}{m_{e} m_{p} a^{3}}
$$

where the Coulomb logarithm $\ln \Lambda \approx 27$ at $1 \mathrm{AU}, m_{p}$ and $m_{e}$ are the proton and electron masses, and $e$ is the magnitude

\footnotetext{
5 Although the Helios measurements were made close to the ecliptic plane, the Parker spiral effect in the inner heliosphere is not as pronounced as it is at $r \geq 1$ AU because of the linear dependence on distance. Thus, the resulting values of $Q_{e}$ at the lowest values of $r$ are relatively insensitive to the choice for $\theta$ (see Fig. $4 b$ ).
} 
of the proton and electron charge (Spitzer 1962). The mean squared interaction speed is given by

$$
a^{2}=\frac{2 k_{\mathrm{B}} T_{p}}{m_{p}}+\frac{2 k_{\mathrm{B}} T_{e}}{m_{e}} .
$$

Note that, as defined above, the quantity $\nu_{p e}$ is a factor of two larger than the equivalently named rate used by Isenberg (1984) and Cranmer et al. (1999). The present definition is more consistent with it being the true rate of temperature equilibration as described by Spitzer (1962). After evaluating many of the constants and using the approximation that $m_{e} \ll m_{p}$, we found

$$
\nu_{p e} \approx 8.4 \times 10^{-9}\left(\frac{n_{e}}{2.5 \mathrm{~cm}^{-3}}\right)\left(\frac{T_{e}}{10^{5} \mathrm{~K}}\right)^{-3 / 2} \mathrm{~s}^{-1} .
$$

This expression gives rise to a rather large mean free path for electron-proton collisions. Depending on whether the mean free path is defined in terms of the solar wind speed $\left(L_{\mathrm{mfp}} \sim\right.$ $\left.u / \nu_{p e}\right)$ or the faster electron thermal speed $\left(L_{\mathrm{mfp}} \sim V_{t h, e} / \nu_{p e}\right)$, this quantity is of order 500-1500 AU at $r=1 \mathrm{AU}$. This should be contrasted with the much smaller mean free path of $\sim 0.5$ $\mathrm{AU}$ for electron-electron self-collisions that maintain the thermal core at 1 AU (see, e.g., Spitzer 1962; Salem et al. 2003).

In addition to Coulomb collisions, there may be other collision-like processes that lead to temperature equilibration and isotropization in the heliosphere. For example, collisionless wave-particle interactions have been suggested for many years as being able to produce these effects (Cuperman \& Harten 1970; Perkins 1973; Dum 1983; Williams 1995; Kel$\log 2$ 2000). If the actual proton-electron temperature equilibration rate is faster than expected, then more heat must go into the protons for them to maintain the known inequality $T_{p}>T_{e}$ in the fast wind. Thus, if the collision rate is anomalously enhanced, the resulting value of $Q_{p}$ would be larger (and $Q_{e}$ would be smaller) than the values computed with weak or nonexistent collisions. We include these effects below by multiplying the collision rates $\nu_{p e}$ and $\nu_{e p}$ by an arbitrary constant $f$.

The electron heat conduction flux affects the energy balance in qualitatively different ways depending on heliocentric distance. Using the empirical fit for $q_{\|, e}(r)$ (eq. [3]) and taking the divergence as shown in equation (9), we found that this term leads to local electron cooling for $r \lesssim 0.75 \mathrm{AU}$, because the radial slope of $q_{\|, e}$ is flatter than $r^{-2}$. In this case, cool plasma at larger radii is conducted inward. At larger distances, though, this term gives local electron heating, since the slope of $q_{\|, e}$ is steeper than $r^{-2}$ and hot plasma is conducted outward. Note, however, that if we had used either the classical Spitzer-Härm heat flux or the collisionless $\alpha_{e}=1.05$ approximation, as shown in Figure $1 b$, then $q_{\|, e}$ would always be slightly steeper than $r^{-2}$ and heat would be conducted outwards at all distances.

\subsection{Results for Proton and Electron Heating}

We solved equations (8) and (9) for the volumetric heating rates $Q_{p}$ and $Q_{e}$ over the range of heliocentric distances covered by the Helios and Ulysses measurements. An interesting aspect of this work is that because the internal energy equations are not being solved for the temperatures-but instead for the heating rates-we can avoid complicated numerical differential equation techniques and use a straightforward algebraic solution for $Q_{p}$ and $Q_{e}$. Even the radial derivatives can be computed analytically from the fits given in $\S 2$. In

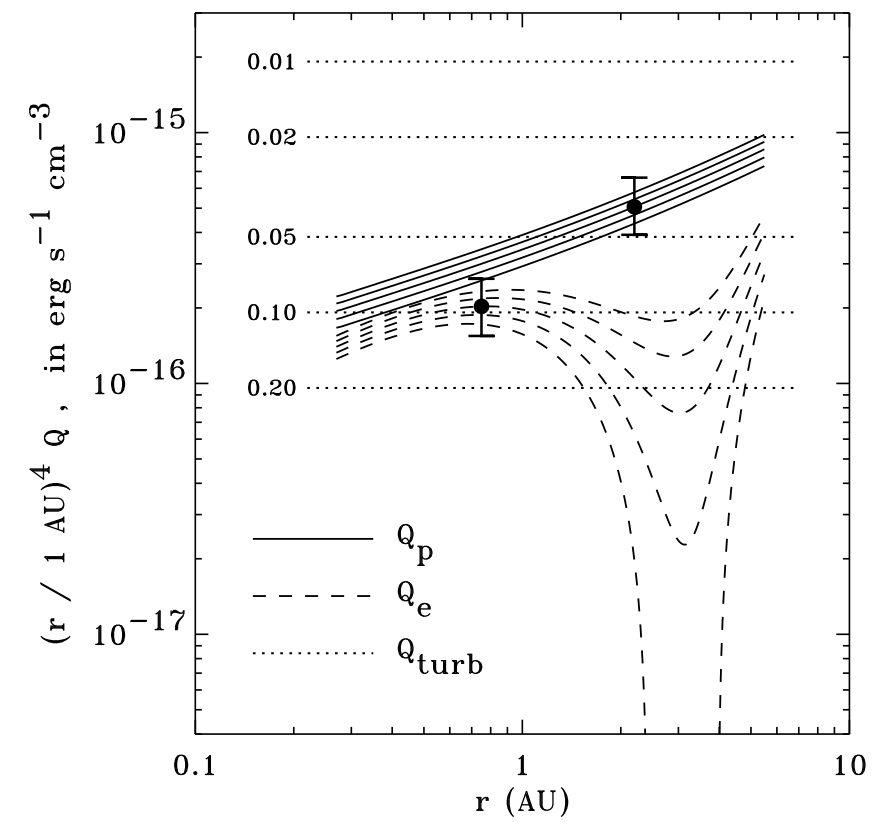

FIG. 2.- Empirically derived heating rates for protons (solid lines) and electrons (dashed lines) in the fast solar wind, with multiple curves showing results for $u=600,650,700,750$, and $800 \mathrm{~km} \mathrm{~s}^{-1}$ (from bottom to top for each set of curves). Shown for comparison is $Q_{\text {turb }}$ (eq. [15]) for five values of $\lambda_{\perp}$ at $1 \mathrm{AU}$ (dotted lines). All heating rates have been multiplied by $(r / 1 \mathrm{AU})^{4}$. Example error bars are given for the $u=700 \mathrm{~km} \mathrm{~s}^{-1}$ case (see text).

practice, however, we computed the quantities to be differentiated on a very fine grid (1000 points between 0.27 and 5.5 AU) and used a standard centered-difference approximation to compute each of the terms in equations (8) and (9).

The internal energy equations were first solved for two cases: (1) standard Coulomb collision rates, with $f=1$, and (2) a completely collisionless heliosphere, with $f=0$. These two cases gave virtually identical results to one another, with relative differences between the heating rates only at the $0.5 \%$ level. The procedure was also carried out several times for a range of assumed (constant) wind speeds $u$ between 600 and $800 \mathrm{~km} \mathrm{~s}^{-1}$. One can see from equation (8) that, in the case of negligible Coulomb collisions, our derived proton heating rate $Q_{p}$ should be linearly proportional to the wind speed $u$. The electron heating rate $Q_{e}$ also increases as the wind speed increases, but because of the heat conduction this is not a purely linear relationship.

Figure 2 shows the quantities $r^{4} Q_{p}(r)$ and $r^{4} Q_{e}(r)$ as a function of radius, rather than the rates themselves, because the latter decrease very steeply with distance. It is easier to see the subtle relative differences between $Q_{p}$ and $Q_{e}$ when the dominant radial variation has been removed. It is clear that the computed values for $Q_{p}$ can be well approximated by power law scaling relations in both radius and wind speed. The following fit was found to be valid to within about $6 \%$ relative accuracy over the full range of distances:

$$
Q_{p} \approx 3.42 \times 10^{-16}\left(\frac{r}{1 \mathrm{AU}}\right)^{-3.5}\left(\frac{u}{700 \mathrm{~km} \mathrm{~s}^{-1}}\right) \mathrm{erg} \mathrm{s}^{-1} \mathrm{~cm}^{-3} .
$$

These scalings compare favorably to similar calculations by Verma et al. (1995) and Vasquez et al. (2007).

Note that it is not possible to fit the electron heating rate $Q_{e}(r)$ with a simple power-law function because of the nonlocal heat conduction. The distances at which $Q_{e}$ decreases rapidly ( $\sim 3 \mathrm{AU})$ are roughly the same as where the "bifurca- 
tion" in $T_{e}$ occurs (see Fig. 1). We cannot conclude whether this is a coincidence or not because of the relatively sparse sampling of the fast wind by Ulysses at these distances, Note also that solving equation (9) gives negative values for $Q_{e}$ at these heights for the smallest wind speed of $600 \mathrm{~km} \mathrm{~s}^{-1}$. The relative impact of the electron heat flux is strong at these distances, and the negative values would be eliminated if the magnitude of $q_{\|, e}$ was smaller by only about $10 \%$. This is certainly within reason, especially since the data used to constrain the fit (Scime et al. 1994) were not specifically taken in the fast solar wind.

Figure 2 also includes representative uncertainty limits, shown as error bars only at two distinct heights in order to distinguish them clearly from the curves showing the variations in $u$. These uncertainty limits reflect the existence of a range of measured values around the mean fitting curves used for $T_{p}$, $T_{e}$, and $q_{\|, e}$. To compute these uncertainty bounds on $Q_{p}$ and $Q_{e}$, we created a set of alternate models in which each of the fits was was multiplied by factors $1.3^{n}$, where $n=-1,0,+1$. The result was a grid of 27 models in which the temperatures and electron heat fluxes were varied up and down (in all combinations) by fiducial $\pm 30 \%$ amounts. The extreme upper and lower limits on $Q_{p}$ and $Q_{e}$ were found for all of these models and are plotted in Figure 2 at the two example distances. As can be seen from Figure 1, the actual spread in the in situ data often reaches - and sometimes exceeds-this fiducial $30 \%$ relative variability level. Thus, it is clear that the results presented here about the proton and electron heating rates depend crucially on the use of the mean radial trends in the plasma parameters. Further work is needed to ensure that these results are valid for the totality of fast solar wind streams. Other approaches to estimating the effect of variability of local parameters on the heating rates include those of Smith et al. (2006a) and Breech et al. (2008).

It is useful to compare the empirically derived heating rates to those expected from a von Kármán similarity analysis of the dissipation of an MHD turbulent cascade (e.g., Hossain et al. 1995). There are several pieces of evidence that suggest turbulence to be responsible for the in situ plasma heating (see, e.g., Coleman 1968). The most direct evidence is the fact that $T_{p}$ in the solar wind is positively correlated with the overall amplitude of the turbulent fluctuations (Grappin et al. 1990; Vasquez et al. 2007). A more thorough discussion of the expected turbulent heating rate is given by, e.g., Breech et al. (2008, 2009). Roughly, though, we can estimate this rate as

$$
Q_{\mathrm{turb}} \approx \frac{\rho\left(Z_{+}^{2} Z_{-}+Z_{-}^{2} Z_{+}\right)}{\lambda_{\perp}}
$$

where the mass density $\rho \propto r^{-2}$ and the transverse correlation length $\lambda_{\perp} \propto r^{1 / 2}$. Note that the heating rate $Q_{\text {turb }}$ depends on the cross helicity of the fluctuations, which is defined by the ratio $\sigma_{c}=\left(Z_{+}^{2}-Z_{-}^{2}\right) /\left(Z_{+}^{2}+Z_{-}^{2}\right)$, and for highly Alfvénic states where $\sigma_{c} \rightarrow \pm 1$, we see that $Q_{\text {turb }} \rightarrow 0$.

The above phenomenological form for the turbulent heating rate has been found to be consistent with numerical simulations of strong MHD turbulence in a background magnetic field (e.g., Dobrowolny et al. 1980; Hossain et al. 1995; Zhou \& Matthaeus 1990; Matthaeus et al. 1999a; Oughton et al. 2001; Dmitruk et al. 2001, 2002). The success of transport theories in accounting for turbulence properties observed by Voyager, Ulysses, and Pioneer data (Zank et al. 1996; Smith et al. 2001; Breech et al. 2008) also provide empirical support for the use of $Q_{\text {turb }}$ in the form given by equation (15).
The radial scaling $\lambda_{\perp} \propto r^{1 / 2}$ has been measured in the heliosphere by, e.g., Bruno \& Dobrowolny (1986) and Smith et al. (2001). The measured Elsasser amplitudes $Z_{ \pm}$exhibit a complex radial dependence that depends on various properties, but it is possible to approximate them reasonably well with WKB Alfvén wave action conservation (Zank et al. 1996). Assuming spherical symmetry-which is appropriate for the high-speed wind at high latitudes - this radial dependence is $Z_{ \pm} \propto r^{-1 / 2}$. Putting these together we find that $Q_{\text {turb }} \propto r^{-4}$, which explains how the above approximations lead to the corresponding curves in Figure 2 being constant (see also Dmitruk et al. 2002; Cranmer \& van Ballegooijen 2005).

To normalize $Q_{\text {turb }}$ to the values shown in Figure 2, we applied measured plasma properties at $1 \mathrm{AU}$, such as the proton number density $\left(2.5 \mathrm{~cm}^{-3}\right)$ and the Elsasser amplitudes corresponding to outwardly propagating $\left(Z_{+} \approx 61 \mathrm{~km} \mathrm{~s}^{-1}\right)$ and inwardly propagating $\left(Z_{-} \approx 26 \mathrm{~km} \mathrm{~s}^{-1}\right)$ Alfvén waves. The latter two quantities combine to give a mean Elsasser amplitude $Z \approx 47 \mathrm{~km} \mathrm{~s}^{-1}$ at $1 \mathrm{AU}$ and a normalized cross helicity $\sigma_{c} \approx 0.7$ (see, e.g., Bavassano et al. 2000; Dasso et al. 2005; Cranmer \& van Ballegooijen 2005; Breech et al. 2008, 2009). The remaining free parameter in equation 15 is the value of the transverse correlation length at $1 \mathrm{AU}$, which we vary between 0.01 and $0.2 \mathrm{AU}$. Figure 2 shows that the empirical heating rates for the fast wind are consistent with $\lambda_{\perp} \approx 0.02$ 0.1 AU. Breech et al. (2008) found that this same range of correlation lengths applies to the fast wind at $1 \mathrm{AU}$ as well. ${ }^{6}$

Figures 3 and 4 show the ratio of proton heating to the total, i.e., $Q_{p} /\left(Q_{p}+Q_{e}\right)$, for a number of different calculations. This fraction is denoted $f_{p}$ by Breech et al. (2009). In all panels, a baseline model is shown for comparison that was computed with $u=700 \mathrm{~km} \mathrm{~s}^{-1}$ and the standard choices for other parameters as described above. Figure $3 a$ shows the dependence of this ratio on the assumed value of the wind speed. The ratio at $r \lesssim 1$ AU appears to be insensitive to the wind speed because both $Q_{p}$ and $Q_{e}$ vary linearly with $u$ at these distances (see also Fig. 2). The increased spread in the ratio at larger distances is the result of the electron heat conduction having a larger relative impact on $Q_{e}$ as the wind speed changes.

Figure $3 b$ shows the dependence of the proton-to-total heating ratio on varying several other assumptions of the standard model (all keeping $u=700 \mathrm{~km} \mathrm{~s}^{-1}$ ). We computed trial versions of the proton temperature fitting curve with the standard Ulysses measurements replaced by either the lower or upper limit measurements $\left(T_{\text {small }}\right.$ or $T_{\text {large }}$ as described in $\left.\S 2\right)$. We also tried replacing the fitting curve for the electron heat conduction flux (eq. [3]) with the simple collisionless expression (eq. [7]) and a constant coefficient $\alpha_{e}=1.05$. This was seen in Figure $1 b$ to possibly be a reasonable description of the measured heat fluxes.

It is noteworthy that the use of the collisionless model for $q_{\|, e}$ shows a rather extreme departure from the other results, with the ratio $Q_{p} /\left(Q_{p}+Q_{e}\right)$ monotonically decreasing as a function of increasing distance. This difference arises because the collisionless heat flux remains steeper than $r^{-2}$ in the inner heliosphere, and thus its divergence (see the last term in eq. 9]) gives a positive contribution to the electron heating at all distances. In a sense, the use of the collisionless heat flux

\footnotetext{
6 Multispacecraft measurements (e.g., Matthaeus et al. 2005) show the measured correlation scale at $1 \mathrm{AU}$ to be somewhat smaller than the values in Figure 2. This can be reconciled by using a value of order $0.1-0.2$ for the von Kármán proportionality constant in equation [15, instead of the value of 1 assumed here (see also Breech et al. 2009).
} 

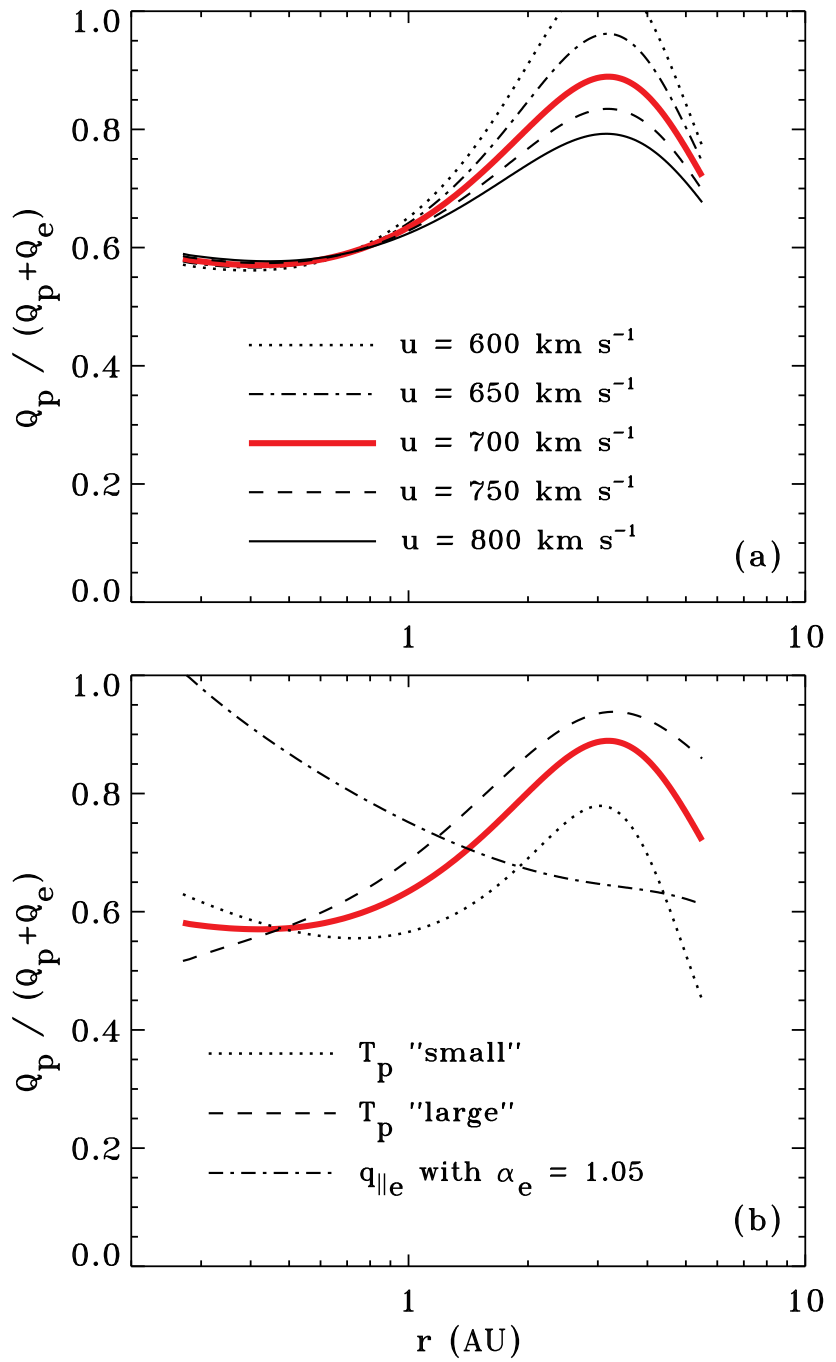

FIG. 3.- Ratios of the proton heating rate to the total (proton + electron) heating rate. The standard model with wind speed $u=700 \mathrm{~km} \mathrm{~s}^{-1}$ is shown in both panels (thick red line). (a) Variation of the wind speed between 600 and $800 \mathrm{~km} \mathrm{~s}^{-1}$ in $50 \mathrm{~km} \mathrm{~s}^{-1}$ increments (see labels). (b) Models computed with $T_{\text {small }}$ (dotted line) and $T_{\text {large }}$ (dashed line) for the SWOOPS proton temperatures, and with equation (7) for $q_{\|, e}$ instead of the empirical fit (dot-dashed line).

is consistent with the need for heat to be conducted outwards from heights below our lower boundary at $0.3 \mathrm{AU}$. The existence of this additional conductive heating below $1 \mathrm{AU}$ means that the deposited heating rate $Q_{e}$ need not be as large as it would be otherwise, and thus the protons end up contributing more to the total heating. On the other hand, when using the least-squares fit to the measured heat fluxes, the slope of $q_{\|, e}$ is flatter than $r^{-2}$ in the inner heliosphere and conduction acts to cool the electrons at these distances. The derived value of $Q_{e}$ must then be larger, which gives the rough equipartition between protons and electrons. This is consistent with a more local deposition of heat to the electrons that conducts both up and down from some point around $0.75 \mathrm{AU}$. We should emphasize, however, that the collisionless expression is based on relatively simple theoretical scalings and it is far from being a rigorous "prediction" for the electron heat flux in the solar wind. The discrepancy seen in Figure $3 b$ suggests that the use of a constant $\alpha_{e}$ coefficient in equation (7) should probably not be considered a robust approximation.

Figure $4 a$ shows the result of varying the Coulomb collision
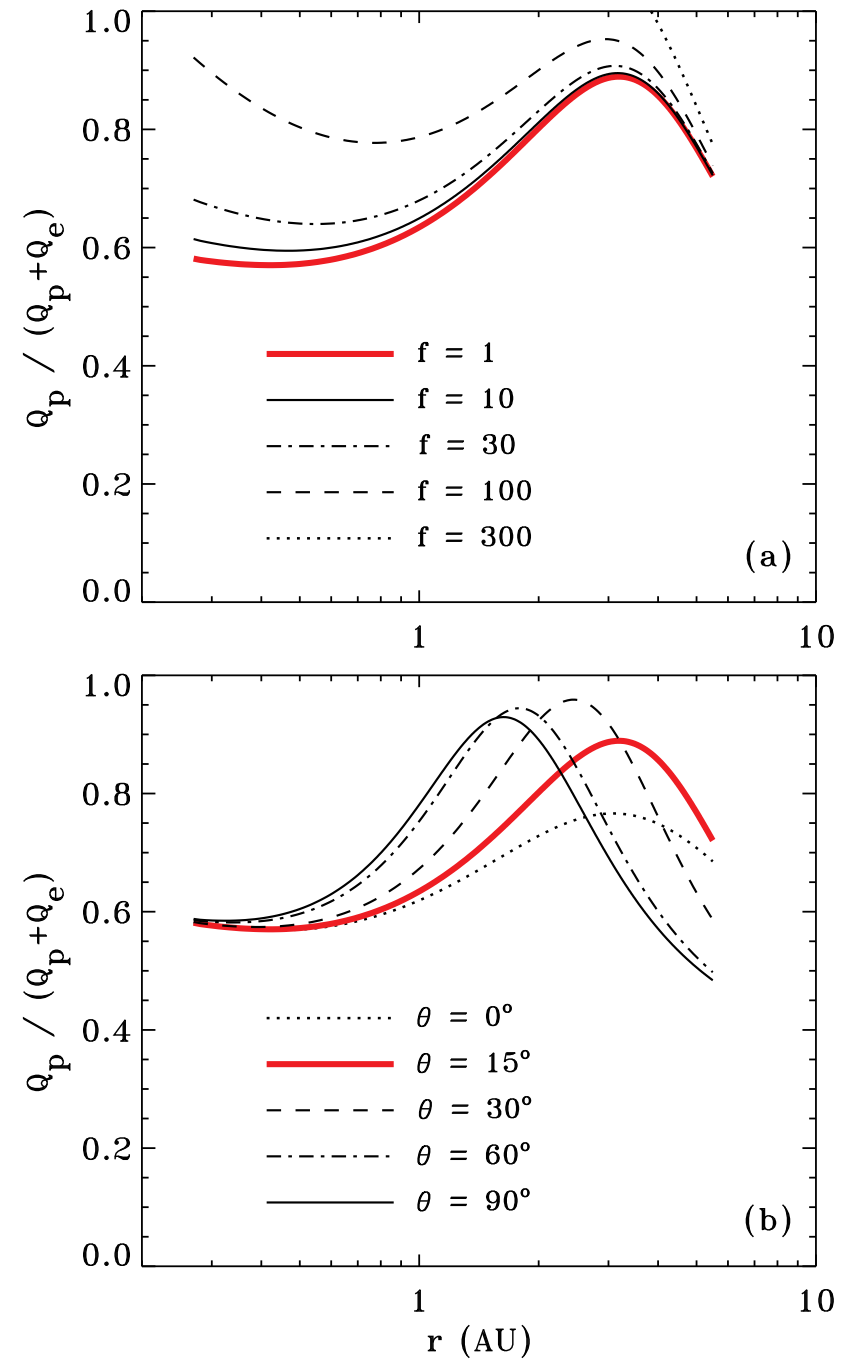

FIG. 4.- Same as Figure 3, but with other parameters varied. (a) A series of models with anomalously strong Coulomb collisions, with a range of constant multipliers $f$ to the classical collision frequency (see labels). (b) Models computed over a range of colatitudes $\theta$ in the heliosphere, which affects the Parker spiral angle $\Phi$ (see eq. [10]). The standard model with wind speed $u=700 \mathrm{~km} \mathrm{~s}^{-1}$ is shown in both panels (thick red line).

rate by multiplying $\nu_{p e}$ (eq. [13]) by a range of constant multipliers $f$. As mentioned above, the curves for $f=0$ and $f=1$ would be indistinguishable and the former is not shown. As $f$ is increased, collisions attempt to equalize $T_{p}$ and $T_{e}$ at a faster rate. The fraction of heat going to the protons must increase in order to maintain the specified $T_{p}>T_{e}$. Note, however, that above a certain value of $f$ there would need to be net electron cooling, or essentially $Q_{p}>\left(Q_{p}+Q_{e}\right)$, in order to maintain the measured temperature difference. This allows us to rule out values of $f$ larger than about 200 over most heliocentric distances. Figure $4 b$ shows how the relative heating changes as the assumed colatitude $\theta$ (and thus the Parker spiral angle $\Phi)$ is varied. Models computed closer to the ecliptic plane exhibit a local maximum in $Q_{p} /\left(Q_{p}+Q_{e}\right)$ at smaller heliocentric distances.

Given the uncertainties in many of the input parameters to these models, the quantitative results regarding the relative partitioning of $Q_{p}$ and $Q_{e}$ are also somewhat uncertain. However, there does appear to be a preponderance of evidence for the validity of two qualitative statements: (1) In the inner heliosphere $(r<1 \mathrm{AU})$, there appears to be rough equipartition 
between proton heating and electron heating. (2) As heliocentric distance is increased from 0.3 to $5 \mathrm{AU}$, the relative fraction of proton heating increases to noticeably dominate the total heating rate.

\section{EXPECTATIONS FROM LINEAR ALFVÉN WAVE DAMPING}

It is illustrative to compare the empirically derived partitioning between proton and electron heating with theoretical predictions. As a starting point for future work in this direction, we computed some extremely simple estimates for the wavenumber dependence of a turbulent power spectrum of Alfvénic fluctuations. These were then coupled to a general Alfvén wave dispersion relation that allowed us to compute the contributions to proton and electron heating. Although it is well-known that strong MHD turbulence is far from "wavelike" (i.e., one might expect that a coherent wave survives for only about one period before nonlinear processes transfer its energy to smaller scales), there is a long history of using damped linear wave theory to study the small-scale dissipation of a cascade (see, e.g., Eichler 1979; Quataert 1998; Leamon et al. 1999; Quataert \& Gruzinov 1999; Marsch \& Tu 2001; Cranmer \& van Ballegooijen 2003; Gary \& Borovsky 2004, 2008). A typical justification of this approach is that the amplitudes of magnetic fluctuations in the dissipation range tend to be extremely small $\left(\left\langle\delta B^{2}\right\rangle \ll B_{0}^{2}\right)$; see also Spangler (1991) and Miller et al. (1996).

We assumed that the wavenumber dependence of the power spectrum of magnetic fluctuations $P_{B}$ scales as $k^{-7 / 2}$, where $k$ is the magnitude of the wavenumber. The power $P_{B}(k)$ is defined such that its integral over the full (three-dimensional) wavenumber space gives the total magnetic energy density of fluctuations. The exponent of $-7 / 2$ corresponds to an exponent of $-3 / 2$ for a corresponding one-dimensional isotropic spectrum (i.e., the latter measuring power in spherical shells in $k$-space). This exponent has been proposed for various theories of MHD turbulence (e.g., Iroshnikov 1964; Kraichnan 1965; Nakayama 2001). This value is also close to the exponent of $-10 / 3$ that has been predicted for a purely perpendicular cascade having a one-dimensional power spectrum of $k_{\perp}^{-5 / 3}$ (see Kolmogorov 1941; Fyfe et al. 1977; Higdon 1984; Goldreich \& Sridhar 1995; Cho \& Vishniac 2000; Boldyrev 2005; Horbury et al. 2008).

We also assumed that the above wavenumber dependence applies in restricted ranges of $\theta_{k B}$, which is defined as the angle between the wavenumber vector and the background magnetic field direction. Specifically, we constructed three models with different angular distributions of wave power: (1) a "slab" spectrum, with nearly parallel-propagating waves filling the region $0 \leq \theta_{k B} \leq 5^{\circ}$, (2) a "two-dimensional" (2D) spectrum, with nearly perpendicularly-propagating waves filling the region $85^{\circ} \leq \theta_{k B} \leq 90^{\circ}$, and (3) an isotropic spectrum with all values of $\bar{\theta}_{k B}$ having the same wavenumber dependence.

The second case given above-i.e., a turbulent cascade mainly in the $k_{\perp}$ direction-uses a range of angles that is actually quite broad in comparison to theoretical expectations of the so-called "critical balance" proposed by Goldreich \& Sridhar (1995). This condition is defined by an equivalence between the time scales of Alfvén wave propagation along the field and nonlinear energy transfer perpendicular to the field. According to Goldreich \& Sridhar (1995), the majority of the power in strong MHD turbulence should have angles between the critical balance angle $\theta_{\text {crit }}$ and $90^{\circ}$. At large perpendicular wavenumbers $k_{\perp}$ (i.e., at the onset of kinetic Alfvén wave dispersion and substantial Landau damping), we estimated that $\theta_{\text {crit }}$ decreases slightly from approximately $89.8^{\circ}$ at $0.3 \mathrm{AU}$ to $88.9^{\circ}$ at $5 \mathrm{AU}$. These values were computed from the existing models of Cranmer \& van Ballegooijen $(2003,2005)$ and the measured plasma parameters given in $\S 2$.

We computed the proton and electron heating rates for each kind of spectrum using the quasi-linear framework developed by Quataert (1998), Quataert \& Gruzinov (1999), Marsch \& Tu (2001), and Cranmer \& van Ballegooijen (2003). The heating rates are given by integrals over vector wavenumber $\mathbf{k}$ of the form

$$
Q_{s}=\rho \int d^{3} \mathbf{k} P_{\mathrm{tot}}(\mathbf{k}) 2 \omega_{i, s}
$$

where $s=p, e$ denotes the particle type of interest and $P_{\text {tot }} \approx$ $2 P_{B}$ is the total energy spectrum of fluctuations. The speciesdependent amplitude damping rates $\omega_{i, s}$ were defined by Cranmer \& van Ballegooijen (2003) to be weighted by Landau/cyclotron resonance functions that take account of the particle kinetic motions. For a proton-electron plasma, the sum $\omega_{i, p}+\omega_{i, e}$ gives the absolute value of the total linear damping rate (i.e., the imaginary part of the wave frequency). The resonance functions depend on the dispersive properties of the Alfvén waves, which were computed using the warmplasma Vlasov-Maxwell dispersion code of Cranmer \& van Ballegooijen (2003). These functions also depend on the shape of the particle velocity distributions, which were assumed here to be Maxwellian.

In order to compute the relative heat dissipated by the protons versus that dissipated by the electrons, we did not need to specify an absolute normalization for the power spectrum. However, in practice, it was useful to cut off the spectrum at low wavenumbers using an "outer scale" lower limit $k_{\text {out }}$ that scaled inversely with the turbulent correlation length discussed above; i.e., $k_{\text {out }} \propto \lambda_{\perp}^{-1} \propto r^{-1 / 2}$. The upper limits for the parallel and perpendicular wavenumbers $\left(k_{\|}, k_{\perp}\right)$ were found numerically by the Vlasov-Maxwell code as locations where the Alfvén-wave solution branches ceased to exist (see Stix 1992; Cranmer \& van Ballegooijen 2003).

The dispersive properties of Alfvén waves depend strongly on the value of the plasma $\beta$, which is defined here as the ratio of proton plasma pressure to the magnetic pressure (see Gary \& Borovsky 2004, 2008). For the high-latitude heliocentric distances considered here, we used a very simple monopolar scaling for the magnetic field strength,

$$
B_{0}(r) \approx 2.5 \times 10^{-5}\left(\frac{r}{1 \mathrm{AU}}\right)^{-2} \mathrm{G}
$$

and $\beta=8 \pi n_{p} k_{\mathrm{B}} T_{p} / B_{0}^{2}$. Using the empirically constrained densities and temperatures discussed above, the value of $\beta$ increases monotonically from 1.1 (at $0.3 \mathrm{AU}$ ) to about 37 (at $5 \mathrm{AU})$. Because calculating the dispersive properties of the waves on a fine two-dimensional grid in wavenumber is computationally expensive, we created only 7 grids having $\beta=1$, $1.8,3.4,6.4,12,22$, and 40 . We computed full runs of $Q_{p}$ and $Q_{e}$ versus heliocentric distance using each of the 7 grids, and then we interpolated between these results (using the empirical plasma $\beta$ at each distance) in order to determine the appropriate solutions.

Figure 5 shows the results of this procedure for the slab, $2 \mathrm{D}$, and isotropic spectra, and we compare the proton heating fractions $Q_{p} /\left(Q_{p}+Q_{e}\right)$ to the standard empirical curve from Figures 3 and 4 . The curve denoting the slab spectrum 


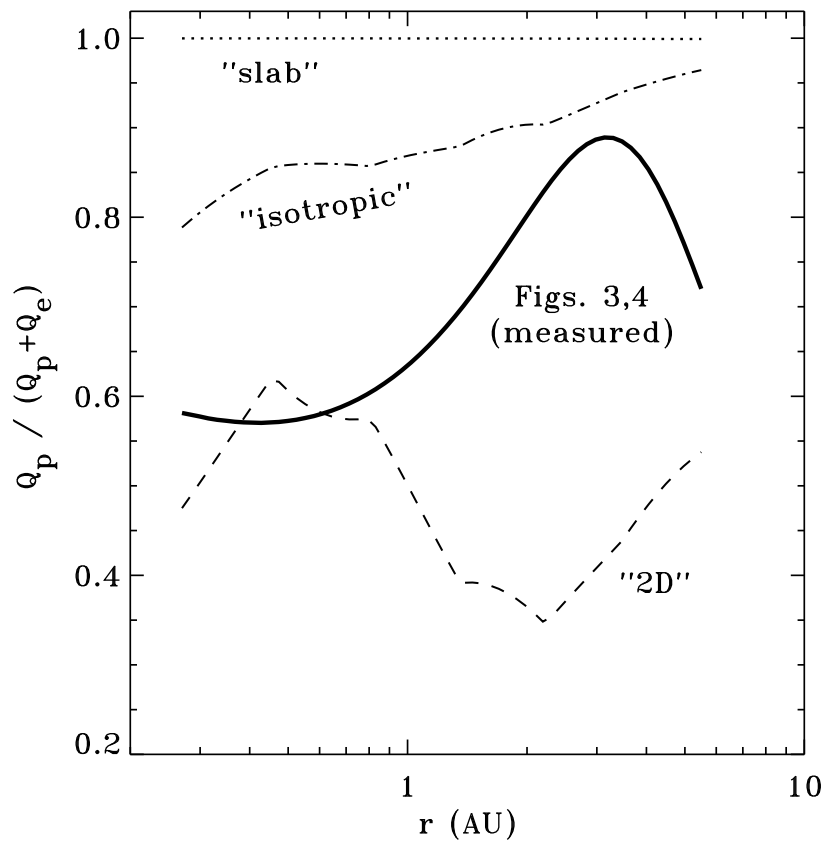

FIG. 5.- Comparison of the standard empirical proton-to-total heating ratio (solid line) to theoretical ratios computed from the linear damping of Alfvén wave spectra: parallel-propagating "slab" modes only (dotted line), perpendicular "2D" modes only (dashed line), and an isotropic spectrum (dot-dashed line)

is relatively simple to understand. In a collisionless plasma, parallel-propagating Alfvén waves dissipate primarily by cyclotron resonance with positive ions (see Hollweg \& Isenberg 2002). For the values of $\beta$ used in the warm-plasma dispersion code, the largest wave frequency for parallel-propagating waves was only about 0.3 times the local proton gyrofrequency. Thus, since the model did not contain any other ions, only the protons felt the cyclotron resonance and were responsible for almost all of the dissipation. There was some extremely weak Landau damping in the slab model that contributed about $0.1 \%$ of the total heating to the electrons.

The curve denoting the 2D spectrum (i.e., fluctuations perpendicular to the background magnetic field) always shows a roughly equal partitioning of energy between protons and electrons. However, the partition fraction oscillates slightly up and down several times between 0.3 and 5 AU. These oscillations do not seem to be numerical artifacts, and their origin is not entirely clear. For the range of plasma $\beta$ found in these models, there is never a single physical process that dominates the collisionless damping of highly oblique Alfvén waves. The mechanisms of electron Landau damping, proton Landau damping, and proton transit-time damping are all of comparable magnitude (e.g., Gary \& Borovsky 2004, 2008). Thus, we refrain from attributing the mild variations around the mean equipartitioning $\left(Q_{p} \approx Q_{e}\right)$ to any specific physical process. We propose to study this complex interplay of collisionless dissipation mechanisms in future work. Finally, the curve denoting an isotropic fluctuation spectrum tends gives an intermediate amount of heat partitioning between the slab and $2 \mathrm{D}$ cases, as one would expect.

By comparing the theoretical and empirical curves in Figure 5 , we can make some preliminary suggestions about how the anisotropy of the actual turbulent power spectrum (or at least its large-wavenumber dissipation range) varies between 0.3 and $5 \mathrm{AU}$. An interpretation based on Figure 5 is that the fluctuations are close to $2 \mathrm{D}$ at the smallest heliocentric distances, and that they evolve to be more isotropic as distance is increased. This makes heuristic sense from at least one limited viewpoint: i.e., that as the plasma $\beta$ increases and the effect of the magnetic field becomes less important to the plasma, the turbulence may want to behave in an increasingly hydrodynamic (i.e., isotropic) fashion. Note, however, that this conclusion is predicated on a wide range of assumptions about the models. Most importantly, it depends on the turbulent fluctuations being purely in the Alfvén mode, and not having, say, fast-mode/whistler type dispersive properties. This conclusion also depends on the turbulent fluctuations damping like linear waves, despite our knowledge that nonlinear features such as current sheets may play an important role (Dmitruk et al. 2004).

We should also note that if the fit to the electron heat conduction (eq. [3]) is incorrect, and instead the collisionless expression of Hollweg $(1974,1976)$ is taken to be valid, then the above conclusions may be reversed. A comparison between the heating partition curve that was computed for $\alpha_{e}=1.05$ (from Fig. $3 b$ ) with the theoretical results in Figure 5 would imply that the turbulence evolves from a slab or isotropic spectrum at $0.3 \mathrm{AU}$ to a more $2 \mathrm{D}$ spectrum at larger distances. This serves to emphasize how much our conclusions about proton and electron heating depend on the accurate knowledge of the radial dependence of $q_{\|, e}$ in the fast solar wind.

There has been a great deal of work done to measure the anisotropy of MHD turbulence in the solar wind. Ultimately, the empirical data for both the particle heating and the fluctuation spectra should be combined to provide the tightest possible constraints on theoretical models. At $1 \mathrm{AU}$, the dominant component of the turbulence does seem to be perpendicular or 2D (e.g., Bieber et al. 1996; MacBride et al. 2008). However, when analyzing the radial dependence of the anisotropy, there have been differing answers to some of the key questions. Some studies that compared the power between the minimum and maximum variance directions concluded that the turbulence becomes more isotropic as distance increases (Klein et al. 1991; Horbury et al. 1995). Other studies found that the perpendicular component becomes more dominant at larger distances (Bavassano et al. 1982; Neugebauer 2004). Such opposite conclusions may be the result of mixing together regions that contain different amounts of fast and slow wind (see, e.g., Dasso et al. 2005) or using different ranges of frequency. Also, the variance anisotropy does not necessarily vary in the same way as the spectral (wavenumber) anisotropy. These results may also depend on subtleties of the analysis technique, such as how the minimum-variance direction is interpreted (Smith et al. 2006b; Tessein et al. 2009) or how effects such as intermittency are treated (Bruno et al. 1999).

\section{CONCLUSIONS AND FUTURE PROSPECTS}

The goal of this paper has been to compute empirical estimates for the rates of proton and electron heating in the fast solar wind between 0.29 and 5.4 AU. A key new aspect of this work has been the incorporation of the measured electron heat conduction flux, which dominates the internal energy balance of electrons in much of the heliosphere. We conclude that the protons receive about $60 \%$ of the total plasma heating in the inner heliosphere, and that this fraction increases to approximately $80 \%$ by the orbit of Jupiter. These results are confirmed by the independent analyses of Leamon et al. (1999) and Breech et al. (2009), who found that a similar proton-tototal heating fraction of $\sim 60 \%$ is consistent with theoretical 
models of MHD turbulent dissipation.

Various uncertainties in the measurements affected our results. For example, the rates of proton and electron heating $\left(Q_{p}\right.$ and $\left.Q_{e}\right)$ were calculated from mean radial trends in the data (as exemplified by the least-squares fits given in $\S 2$ ), and they do not describe the substantial measured spread in the data about the mean values. Also, the uncertainty in $Q_{e}$ increases with heliocentric distance because the electron heat conduction flux becomes more important-in a relative sense-as distance increases. Small uncertainties in $q_{\|, e}$ or its radial slope thus have a larger impact on the derived heating rates at distances greater than $\sim 2 \mathrm{AU}$. In any case, the general techniques presented in this paper are applicable to any future measurements that would improve on our current knowledge of the solar wind plasma properties.

It is interesting to compare the interplanetary proton and electron heating rates with those inferred for the solar corona. The observational consensus since the late 1990s has been that coronal holes undergo preferential proton heating in a similar sense as high-speed wind streams in the heliosphere (see, e.g., Kohl et al. 1997, 1998, 2006; Wilhelm et al. 2007). Recently, Figure 6 of Landi \& Cranmer (2009) summarized a range of measurements of $T_{p}$ and $T_{e}$ in polar coronal holes. Although one generally finds $T_{p}>T_{e}$, the measurements of $T_{p}$ and $T_{e}$ do not yet fully overlap with one another in heliocentric distance. Improved measurements are needed in order to better constrain the proton and electron heating rates in the corona. However, given that the corona and the heliosphere are so different in density, Alfvén speed, and plasma $\beta$, it is somewhat surprising that the proton-electron heat partitioning in these regions may be so similar in character.

An improved understanding of the so-called two-fluid nature of solar wind plasma is an important ingredient in produc- ing better quantitative predictions of the heliospheric properties relevant to space weather. Most advanced globalmodeling efforts currently include only a one-fluid treatment of the internal energy equation (e.g., Riley et al. 2001, 2006; Roussev et al. 2003; Tóth et al. 2005; Usmanov \& Goldstein 2006; Feng et al. 2007). It is often assumed that in the highest density (i.e., most strongly collisional) regions of the heliosphere, the departures from thermal equilibrium are unimportant. However, there are several kinds of large-scale effects that depend on how heat is deposited into protons, electrons, and possibly heavy ions as well. For example, if all of the coronal heating goes into electrons, there can be substantially more downward conduction than in the proton-heated case, which would affect the location of the coronal temperature maximum (Hansteen \& Leer 1995) and the dynamical stability of helmet streamers (Endeve et al. 2004). Thus, the eventual inclusion of differences between proton heating and electron heating in global models may be a key to improving their physical realism and predictive accuracy.

The authors would like to thank Adriaan van Ballegooijen and Eliot Quataert for valuable discussions. SRC's work was supported by the National Aeronautics and Space Administration (NASA) under grants NNG04GE77G, NNX06AG95G, and NNX09AB27G to the Smithsonian Astrophysical Observatory. WHM's research was supported by NSF ATM 0752135 (SHINE) and NASA NNX08AI47G (Heliophysics Theory Program). BAB's research was supported in part by an appointment to the NASA Postdoctoral Program at Goddard Space Flight Center, administered by Oak Ridge Associated Universities through a contract with NASA. JCK's research was supported in part by NASA grant NNX08AW07G.

\section{REFERENCES}

Arya, S. \& Freeman, J. W. 1991, J. Geophys. Res., 96, 14183

Bame, S. J., McComas, D. J., Barraclough, B. L., Phillips, J. L., Sofaly, K. J., Chavez, J. C., Goldstein, B. E., \& Sakurai, R. K. 1992, A\&AS, 92, 237

Barakat, A. R., \& Schunk, R. W. 1982, Plasma Phys., 24, 389

Bavassano, B., Dobrowolny, M., Fanfoni, G., Mariani, F., \& Ness, N. F. 1982, Sol. Phys., 78, 373

Bavassano, B., Pietropaolo, E., \& Bruno, R. 2000, J. Geophys. Res., 105, 15959

Bieber, J. W., Wanner, W., \& Matthaeus, W. H. 1996, J. Geophys. Res., 101, 2511

Boldyrev, S. 2005, ApJ, 626, L37

Braginskii, S. I. 1965, Rev. Plasma Phys., 1, 205

Breech, B., Matthaeus, W. H., Cranmer, S. R., Kasper, J., \& Oughton, S. 2009, J. Geophys. Res., in press

Breech, B., Matthaeus, W. H., Minnie, J., Bieber, J. W., Oughton, S., Smith, C. W., \& Isenberg, P. A. 2008, J. Geophys. Res., 113, A08105

Bruno, R., Bavassano, B., Pietropaolo, E., Carbone, V., \& Veltri, P. 1999, Geophys. Res. Lett., 26, 3185

Bruno, R., \& Dobrowolny, M. 1986, Ann. Geophys., 4, 17

Cho, J., \& Vishniac, E. T. 2000, ApJ, 539, 273

Coleman, P. J., Jr. 1968, ApJ, 153, 371

Cranmer, S. R. 2002, Space Sci. Rev., 101, 229

Cranmer, S. R., Field, G. B., \& Kohl, J. L. 1999, ApJ, 518, 937

Cranmer, S. R., \& van Ballegooijen, A. A. 2003, ApJ, 594, 573

Cranmer, S. R., \& van Ballegooijen, A. A. 2005, ApJS, 156, 265

Cuperman, S., \& Harten, A. 1970, ApJ, 162, 315

Dasso, S., Milano, L. J., Matthaeus, W. H., \& Smith, C. W. 2005, ApJ, 635, L181

Dmitruk, P., Matthaeus, W. H., Milano, L. J., Oughton, S., Zank, G. P., \& Mullan, D. J. 2002, ApJ, 575, 571

Dmitruk, P., Matthaeus, W. H., \& Seenu, N. 2004, ApJ, 617, 667

Dmitruk, P., Milano, L. J., \& Matthaeus, W. H. 2001, ApJ, 548, 482

Dobrowolny, M., Mangeney, A., \& Veltri, P. 1980, Phys. Rev. Lett., 45, 144

Dum, C. T. 1983, in Solar Wind Five, ed. M. Neugebauer, NASA CP-2280, 369
Ebert, R. W., McComas, D. J., Elliott, H. A., Forsyth, R. J., \& Gosling, J. T. 2009, J. Geophys. Res., 114, A01109

Eichler, D. 1979, ApJ, 229, 413

Endeve, E., Holzer, T. E., \& Leer, E. 2004, ApJ, 603, 307

Eyni, M., \& Steinitz, R. 1981, ApJ, 243, 279

Feng, X., Zhou, Y., \& Wu, S. T. 2007, ApJ, 655, 1110

Freeman, J. W. 1988, Geophys. Res. Lett., 15, 88

Fyfe, D., Joyce, G., \& Montgomery, D. 1977, J. Plasma Phys., 17, 317

Gary, S. P., \& Borovsky, J. E. 2004, J. Geophys. Res., 109, A06105

Gary, S. P., \& Borovsky, J. E. 2008, J. Geophys. Res., 113, A12104

Goldreich, P., \& Sridhar, S. 1995, ApJ, 438, 763

Goldstein, B. E., et al. 1996, A\&A, 316, 296

Goldstein, M. L., Roberts, D. A., \& Matthaeus, W. H. 1995, ARA\&A, 33, 283

Grappin, R., Mangeney, A., \& Marsch, E. 1990, J. Geophys. Res., 95, 8197 Hansteen, V. H., \& Leer, E. 1995, J. Geophys. Res., 100, 21577

Hellinger, P., Trávníček, P., Kasper, J. C., \& Lazarus, A. J. 2006, Geophys. Res. Lett., 33, L09101

Higdon, J. C. 1984, ApJ, 285, 109

Hollweg, J. V. 1974, J. Geophys. Res., 79, 3845

Hollweg, J. V. 1976, J. Geophys. Res., 81, 1649

Hollweg, J. V., \& Isenberg, P. A. 2002, J. Geophys. Res., 107 (A7), 1147

Horbury, T. S., Balogh, A., Forsyth, R .J., \& Smith, E. J. 1995, Geophys. Res. Lett., 22, 3405

Horbury, T. S., Forman, M., \& Oughton, S. 2008, Phys. Rev. Lett., 101, 175005

Hossain, M., Gray, P. C., Pontius, D. H., Jr., Matthaeus, W. H., \& Oughton, S. 1995, Phys. Fluids, 7, 2886

Iroshnikov, P. S. 1964, Soviet Ast., 7, 566

Isenberg, P. A. 1984, J. Geophys. Res., 89, 6613

Issautier, K., Meyer-Vernet, N., Moncuquet, M., \& Hoang, S. 1998, J. Geophys. Res., 103, 1969

Kasper, J. C., Lazarus, A. J., \& Gary, S. P. 2002, Geophys. Res. Lett., 29, 1839

Kasper, J. C., Lazarus, A. J., \& Gary, S. P. 2008, Phys. Rev. Lett., 101, 261103 
Kellogg, P. J. 2000, ApJ, 528, 480

Klein, L. W., Roberts, D. A., \& Goldstein, M. L. 1991, J. Geophys. Res., 96, 3779

Kohl, J. L., et al. 1997, Sol. Phys., 175, 613

Kohl, J. L., et al. 1998, ApJ, 501, L127

Kohl, J. L., Noci, G., Cranmer, S. R., \& Raymond, J. C. 2006, A\&A Rev., 13, 31

Kolmogorov, A. N. 1941, Dokl. Akad. Nauk SSSR, 30, 301

Kraichnan, R. H. 1965, Phys. Fluids, 8, 1385

Landi, E., \& Cranmer, S. R. 2009, ApJ, 691, 794

Leamon, R. J., Smith, C. W., Ness, N. F., \& Wong, H. K. 1999 , J. Geophys. Res., 104, 22331

Leer, E., Holzer, T. E., \& Flå, T. 1982, Space Sci. Rev., 33, 161

Lopez, R. E., \& Freeman, J. W. 1986, J. Geophys. Res., 91, 1701

MacBride, B. T., Smith, C. W., \& Forman, M. A. 2008, ApJ, 679, 1644

Marino, R., Sorriso-Valvo, L., Carbone, V., Noullez, A., Bruno, R., \& Bavassano, B. 2008, ApJ, 677, L71

Marsch, E. 1999, Space Sci. Rev., 87, 1

Marsch, E., Mühlhäuser, K.-H., Schwenn, R., Rosenbauer, H., Pilipp, W., \& Neubauer, F. M. 1982, J. Geophys. Res., 87, 52

Marsch, E., \& Tu, C.-Y. 2001, J. Geophys. Res., 106, 227

Matteini, L., Landi, S., Hellinger, P., Pantellini, F., Maksimovic, M., Velli, M., Goldstein, B. E., \& Marsch, E. 2007, Geophys. Res. Lett., 34, L20105

Matthaeus, W. H., Dasso, S., Weygand, J. M., Milano, L. J., Smith, C. W., \& Kivelson, M. G. 2005, Phys. Rev. Lett., 95, 231101

Matthaeus, W. H., Dmitruk, P., Oughton, S., \& Mullan, D. 2003, in Solar Wind Ten, AIP Conf. Ser. 679, ed. M. Velli \& R. Bruno (Woodbury, NY: AIP), 427

Matthaeus, W. H., Zank, G. P., Oughton, S., Mullan, D. J., \& Dmitruk, P. 1999a, ApJ, 523, L93

Matthaeus, W. H., Zank, G. P., Smith, C. W., \& Oughton, S. 1999b, Phys. Rev. Lett., 82, 3444

Miller, J. A., LaRosa, T. N., \& Moore, R. L. 1996, ApJ, 461, 445

Nakayama, K. 2001, ApJ, 556, 1027

Neugebauer, M. 1982, Space Sci. Rev., 33, 127

Neugebauer, M. 2004, J. Geophys. Res., 109, A02101

Olsen, E. L., \& Leer, E. 1996, J. Geophys. Res., 101, 15591

Oughton, S., Matthaeus, W. H., Dmitruk, P., Milano, L. J., Zank, G. P., \& Mullan, D. J. 2001, ApJ, 551, 565

Parker, E. N. 1963, Interplanetary Dynamical Processes (New York: Interscience)

Perkins, F. 1973, ApJ, 179, 637

Phillips, J. L., Bame, S. J., Gary, S. P., Gosling, J. T., Scime, E. E., \& Forsyth, R. J. 1995, Space Sci. Rev., 72, 109

Phillips, J. L., \& Gosling, J. T. 1990, J. Geophys. Res., 95, 4217

Pilipp, W. G., Miggenrieder, H., Mühlhäuser, K.-H., Rosenbauer, H., \& Schwenn, R. 1990, J. Geophys. Res., 95, 6305

Quataert, E. 1998, ApJ, 500, 978

Quataert, E., \& Gruzinov, A. 1999, ApJ, 520, 248
Riley, P., Linker, J. A., \& Mikić, Z. 2001, J. Geophys. Res., 106, 15889

Riley, P., Linker, J. A., Mikić, Z., Lionello, R., Ledvina, S. A., \& Luhmann, J. G. 2006, ApJ, 653, 1510

Roussev, I. I., Gombosi, T. I., Sokolov, I. V., Velli, M., Manchester, W., IV, DeZeeuw, D. L., Liewer, P., Tóth, G., \& Luhmann, J. 2003, ApJ, 595, L57

Salem, C., Hubert, D., Lacombe, C., Bale, S. D., Mangeney, A., Larson, D. E., \& Lin, R. P. 2003, ApJ, 585, 1147

Sandbæk, Ø., \& Leer, E. 1995, ApJ, 454, 486

Schwenn, R., \& Marsch, E., eds. 1990, Physics of the Inner Heliosphere (Heidelberg: Springer-Verlag)

Scime, E. E., Badeau, A. E., Jr., \& Littleton, J. E. 1999, Geophys. Res. Lett., 26, 2129

Scime, E. E., Bame, S. J., Feldman, W. C., Gary, S. P., Phillips, J. L., \& Balogh, A. 1994, J. Geophys. Res., 99, 23401

Scudder, J. D., \& Olbert, S. 1979, J. Geophys. Res., 84, 6603

Sittler, E. C., Jr., \& Scudder, J. D. 1980, J. Geophys. Res., 85, 5131

Smith, C. W., Matthaeus, W. H., Zank, G. P., Ness, N. F., Oughton, S., \& Richardson, J. D. 2001, J. Geophys. Res., 106, 8253

Smith, C. W., Isenberg, P. A., Matthaeus, W. H., \& Richardson, J. D. 2006a, ApJ, 638, 508

Smith, C. W., Vasquez, B. J., \& Hamilton, K. 2006b, J. Geophys. Res., 111, A09111

Spangler, S. R. 1991, ApJ, 376, 540

Spitzer, L., Jr. 1962, Physics of Fully Ionized Gases, 2nd ed. (New York: Wiley)

Spitzer, L., Jr., \& Härm, R. 1953, Phys. Rev., 89, 977

Stawarz, J. E., Smith, C. W., Vasquez, B. J., Forman, M. A., \& MacBride, B. T. 2009, ApJ, 697, 1119

Stix, T. H. 1992, Waves in Plasmas (New York: AIP)

Tessein, J. A., Smith, C. W., MacBride, B. T., Matthaeus, W. H., Forman, M. A., \& Borovsky, J. E. 2009, ApJ, 692, 684

Tóth, G., et al. 2005, J. Geophys. Res., 110, A12226

Totten, T. L., Freeman, J. W., \& Arya, S. 1995, J. Geophys. Res., 100, 13

Tu, C.-Y. 1988, J. Geophys. Res., 93, 7

Tu, C.-Y., \& Marsch, E. 1995, Space Sci. Rev., 73, 1

Usmanov, A. V., \& Goldstein, M. L. 2006, J. Geophys. Res., 111, A07101

Vasquez, B. J., Smith, C. W., Hamilton, K., MacBride, B. T., \& Leamon, R. J. 2007, J. Geophys. Res., 112, A07101

Verma, M. K., Roberts, D. A., \& Goldstein, M. L. 1995, J. Geophys. Res., 100,19839

Wilhelm, K., Marsch, E., Dwivedi, B. N., \& Feldman, U. 2007, Space Sci. Rev., 133, 103

Williams, L. L. 1995, ApJ, 453, 953

Zank, G. P., Matthaeus, W. H., \& Smith, C. W. 1996, J. Geophys. Res., 101, 17093

Zhou, Y., \& Matthaeus, W. H. 1990, J. Geophys. Res., 95, 10291 